\title{
Higher order Motional Resonances Spectra of electrons with Non-linear Axial Oscillations in Quadrupole Penning trap
}

\author{
B. M. Dyavappa* \\ Department of Physics, Government College for Women, Kolar, Karnataka, India
}

\begin{abstract}
Experimentally the motional resonances spectra of an electron cloud confined in a quadrupole Penning trap, weakly excited with RF field, which is swept from $1 \mathrm{MHz}$ to $1 \mathrm{GHz}$ was examined. The higher order motional resonances of the electron cloud with non-linear axial oscillations show that they result from couplings in the motional degrees of freedom, which characterize the perturbations in the trap, and cause loss of electrons from the trap. The likely presence of perturbative terms of quadrupole potential is identified by studying the motional resonances spectra and hence the multipole terms are calculated. A periodically forced barrel spring with a steel ball at the free end can be regarded as a model of non-linear oscillator and a periodically forced steel ball hung through a flexible rod, which is deflected alternately toward the two magnets can be regarded as a mechanical model of Duffing oscillator are developed, in analogy to observed higher order motional resonances of the electron cloud excited with different RF field powers.
\end{abstract}

DOI:10.46481/jnsps.2022.371

Keywords: Quadrupole Penning trap, Motional resonance spectrum, quadrupole potential, Duffing oscillator, non-linear oscillator

Article History :

Received: 30 August 2021

Received in revised form: 25 October 2021

Accepted for publication: 02 January 2021

Published: 28 February 2022

(c)2022 Journal of the Nigerian Society of Physical Sciences. All rights reserved. Communicated by: W. A. Yahya

\section{Introduction}

The motional resonances spectra of electrons contain the fundamental eigen frequencies, viz. the modified cyclotron frequency $\left(f_{c}^{\prime}\right)$, magnetron frequency $\left(f_{m}\right)$, axial oscillation frequency $\left(f_{z}\right)$ and their linear combinations in Penning trap [1,2]. These frequencies can be measured by resonant excitation of the motion of electrons with RF-field applied to the trap electrode. The linear combinations of the fundamental frequencies and their harmonics become visible in the spectrum of motional resonances, which also depend on the power of the RF field [3]. The pure cyclotron frequency $\left(f_{c}\right)$ can be obtained

${ }^{*}$ Corresponding author tel. no: +919110854782

Email address: dyavappabm@gmail .com (B. M. Dyavappa ) from direct excitation of the side band frequency $\left(f_{c}^{\prime}+f_{m}\right)$. In this paper investigations on motional resonances of an electron cloud probed from the radio frequency of $1 \mathrm{MHz}$ to $1 \mathrm{GHz}$ are presented, which helps in characterizing the instabilities of the electron plasma in the trap. The non-linear higher order motional resonances of the electron cloud show that they result from couplings in the motional degrees of freedom that characterize the perturbations in the trap $[4,5,6]$.

\section{Theory}

The general form of the potential in Penning trap can be written as $[7,8,9,10,11]$ 


$$
\phi(r, \theta)=\phi_{0} \sum_{0}^{n} C_{n}\left(\frac{r}{r_{0}}\right)^{n} P_{n}(\cos \theta)
$$

The presence of higher order terms in the potential due to perturbations cause couplings among the motional degrees of freedom of electrons, and result in motional resonances which occur at certain linear combinations of fundamental frequencies in addition to the characteristic fundamental Eigen frequencies and their harmonics $[7,8,9]$. Thus at these frequencies in motional resonances spectra, we may express the relation among the pure eigen frequencies as $[1,2,9,10,11]$

$$
n_{c}^{\prime} f_{c}^{\prime}+n_{m} f_{m}+n_{z} f_{z}=0
$$

where $n_{c}^{\prime}, n_{m}, n_{z}$ are integers, there are many possible sets of integer triplets $\left(n_{c}^{\prime}, n_{m}, n_{z}\right)$ which can be predicted using MAT Lab Code, but only a few are observed in motional resonances spectra. We know that the eigen frequencies are given by $[7,8$, $9,10,11]$

$$
\begin{aligned}
& f_{c}^{\prime}=\frac{f_{c}+\sqrt{f_{c}^{2}-2 f_{z}^{2}}}{2}, f_{m}=\frac{f_{c}-\sqrt{f_{c}^{2}-2 f_{z}^{2}}}{2}, \\
& f_{c}=f_{c}^{\prime}+f_{m}=\frac{q B}{2 \pi m}
\end{aligned}
$$

Let

$$
A=2\left(n_{c}^{\prime}-n_{m}\right)^{2}+4 n_{z}^{2}, B=-2\left(n_{c}^{\prime}+n_{m}\right) n_{z}, C=4 n_{c}^{\prime} n_{m},(4)
$$

then

$$
\begin{aligned}
& A \omega_{z}^{2}-2 B \omega_{c} \omega_{z}+C \omega_{c}^{2}=0 \\
& \therefore K=\frac{B \pm \sqrt{B^{2}-A C}}{A} .
\end{aligned}
$$

Here $K=\frac{\omega_{z}}{\omega_{c}}=\frac{f_{z}}{f_{c}}, f_{c}>\sqrt{2} f_{z}, K_{\max }=1 / \sqrt{2}$, where $K$ is a constant related to the integers $n_{c}^{\prime}, n_{m}$, and $n_{z}$. For stable trapping condition, $\omega_{c}>\sqrt{2} \omega_{z}$ and $K_{\max }=1 / \sqrt{2}=0.7071$ or $0<K<1 / \sqrt{2}$. One can compute numerically the integer triplet $\left(n_{c}^{\prime}, n_{m}, n_{z}\right)$ sets for a given value of $\mathrm{K}$, and hence a list of possible integer triplets for different values of $\mathrm{K}$. Motional instabilities may occur at frequencies that satisfy 2 for any particular value of $K<1 / \sqrt{2}$.

Table 1: Different values of $\mathrm{K}$ for different magnetic fields and storage voltages

\begin{tabular}{ccc}
\hline $\mathrm{B}\left(10^{-4} T\right)$ & $\mathrm{K}(2 \mathrm{~V})$ & $\mathrm{K}(10 \mathrm{~V})$ \\
\hline 97 & 0.069878 & 0.210543 \\
\hline 258 & 0.032437 & 0.072532 \\
\hline
\end{tabular}

An expression of the potential of the form given by equation (1) in a Fourier series restricts the integer triplets as $[7,8,9,10]$

$$
\left|n_{c}^{\prime}\right|+\left|n_{m}\right|+\left|n_{z}\right| \leq n
$$

This equation indicates that the perturbations at least of order $n$ are present. However all of these perturbations may not cause instabilities.

\section{Experimental Procedure}

The quadrupole Penning trap is designed with threeelectrode infinite hyperboloid revolution of structure, which consists of two end-cap electrodes and a ring electrode. The equation of hyperbola of ring electrode is $\frac{r^{2}}{r_{0}^{2}}-\frac{z^{2}}{z_{0}^{2}}=+1$ and that of two similar end - cap electrodes are given by $\frac{r^{2}}{r_{0}^{2}}-\frac{z^{2}}{z_{0}^{2}}=-1$. The dimensions of Quadrupole Penning trap are designed such that $r_{0}=\sqrt{2} z_{0}=7 \mathrm{~mm}$, where $r_{0}$ is the inner radius of the ring electrode in the radial plane and $z_{0}=5 \mathrm{~mm}$ is half of the vertical distance between the two end-cap electrodes. A thoriated - Tungsten filament is used to generate electrons by thermionic emission through the passage of small $6 \mathrm{~V} \mathrm{dc}$ current. We generate magnetic field using an electromagnet by passing a dc current of $0-20$ A through a pair of coils of wire separated by $11.5 \mathrm{~cm}$. Magnetic field is generated by electromagnetic induction and it can be measured using Hall probe and magnetic field at the trap centre measured from cyclotron frequency from Motional resonances spectra is very accurate is mentioned here. The electrons are trapped in ion trap at a vacuum pressure range of $10^{-6}$ to $10^{-10}$ torr range created by turbo molecular pump with backup rotary vane pump and ion pump. High vacuum of $10^{-7}$ torr can be created and maintained by turbo molecular pump. An ion pump is used to create ultra high vacuum of up to $10^{-10}$ torr [11].

The arrangement to measure the motional resonances of trapped cloud of electrons in Penning trap is described in this section. The couplings in the motional degrees of freedom of the electron cloud are determined by probing the confined cloud of electrons, through exciting the additional modes of oscillations by external RF field applied through an external antenna as shown in Figure 1. We fed RF energy into the trap using a small antenna placed into a narrow hole of the ring electrode and it is isolated from the ring using a ceramic washer. The antenna is basically a co-axial cable of copper wire; the outer insulating cover has been removed partly and inserted into a narrow hole made in the ring electrode. The insulator separating the outer metal sheath and the inner copper wire is not an UHV compatible material, but we have created a vacuum of $10^{-9}$ torr without much difficulty, beyond which one may have to use an alternative material. The RF generator switched to sweep mode and is used to feed RF energy into the trap through the antenna.

The RF power is kept very low of the order of a few / $\mathrm{X}$ to weakly probe the motion of the trapped electron cloud. If the RF power is kept high, then it will resonantly drive the trapped electron cloud in the trap and more electrons are lost. The constant signal strength of resonance absorption signal of electrons in the absence of external excitations is recorded, and the detection cycle is repeated continuously. The constant signal height of electrons serves as a baseline of reference for the motional resonance spectrum measurements. When the electrons are excited by continuously sweeping the RF field, the motional frequencies of electrons respond to the external RF field at an appropriate step of frequency, consequently some of the electrons gain enough energy to escape the trap, consequently the signal strength is reduced and appear as dips, whose strength is di- 


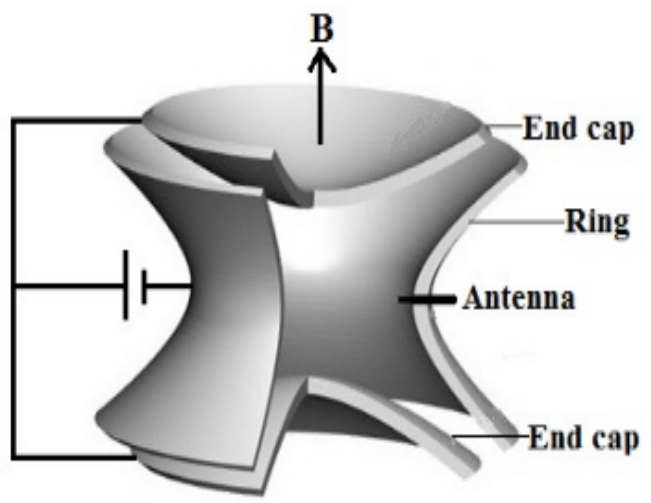

Figure 1: Quadrupole Penning trap with antenna to feed RF field

rectly proportional to the total number of electrons lost from the trap. The data of signals of electrons are continuously acquired and recorded using a Lab VIEW program written in a computer, which yields the desired motional spectrum of trapped electrons. A personal computer installed with Lab VIEW program is used for data acquisition and control trap system using a multifunction NI DAQ card. It controls, monitors and synchronizes four tasks viz. generates the trap potential and timing, controls the electron loading, and controls the RF Sweep and data acquisition. The Motional Resonance Control and Acquisition (MRCA) Lab VIEW program runs all the tasks simultaneously and are performed in a times share method and all tasks are synchronized at the beginning. All the four modules of the program and the tasks are described in detail below:

Trap potential timing sequence: The ring voltage is timed so that it is suitable to trap and detection, to meet the requirements of motional resonances and the time dependence of the voltage is controlled by the Lab VIEW program called Motional Resonance Control and Acquisition (MRCA) using computer. The time measurement cycle has mainly 6 stages as shown in Fig.2 $[1,6,11,12]$.

(i) Loading control: The electron loading can be controlled by controlling filament current or the negative bias voltage provided by a DC power supply applied between the filament and the closer end cap. The electron loading is turned OFF by reducing the bias or brought close to zero. The bias is turned ON during the creation time $\left(t_{1}\right)$, for a short while of about $100 \mathrm{~ms}$ and then turned OFF till the first detection cycle is over.

(ii) Sweep control: A radio frequency generator operated in the sweep mode is used for generating RF field which is fed through the antenna. The sweep is done in two frequency ranges, the first range is from $1-100 \mathrm{MHz}$ in steps of $0.1 \mathrm{MHz}$ and the second range is $100-1000 \mathrm{MHz}$ in steps of $1 \mathrm{MHz}$.

(iii) Data acquisition: The trap potential with the pre-defined time cycle begins; the data is acquired continuously and sampled at a rate of 1000 samples per second using the Lab VIEW program developed in computer and the data obtained is plotted in Origin.

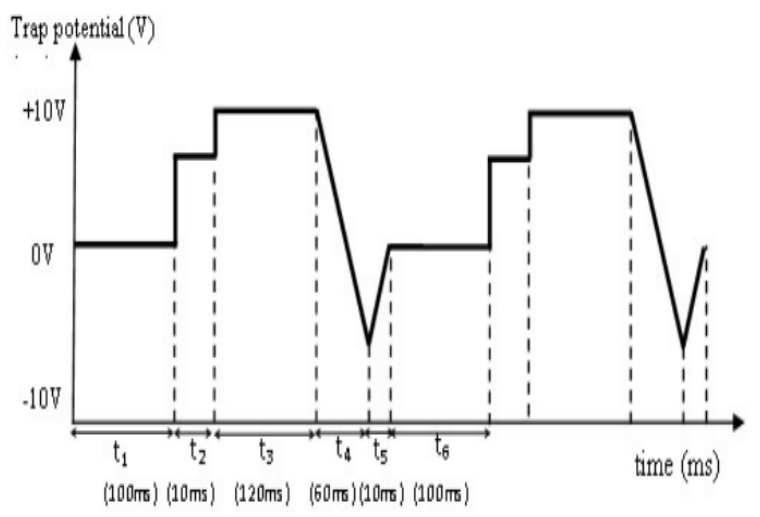

Figure 2: Trap potential timing sequence, $t_{1}$ : Creation time $(100 \mathrm{~ms}), t_{2}$ : Cooling time $(10 \mathrm{~ms}), t_{3}$ : Storage time $(120 \mathrm{~ms}), t_{4}$ : Detection time $(60 \mathrm{~ms}), t_{5}$ : Kick out time $(10 \mathrm{~ms}), t_{6}$ : Waiting time $(100 \mathrm{~ms})$.

\section{Results and Discussion}

The motional resonances spectra of trapped electrons contain the fundamental oscillation frequencies $f_{c}^{\prime}, f_{m}, f_{z}$, in addition some of their linear combinations, satisfying the equation $n_{c}^{\prime} f_{c}^{\prime}+n_{m} f_{m}+n_{z} f_{z}=C$ and multiples of these frequencies $n_{c}^{\prime} f_{c}^{\prime}$, $n_{m} f_{m}$, and $n_{z} f_{z}$. We have carried out measurements close to the stability limit at magnetic fields of $97 \mathrm{G}$ and $258 \mathrm{G}$.

(i) RF field power dependence: When the power of RF field is $-10 \mathrm{dBm}$ then the trapped cloud of electrons is more unstable. More resonances dips are observed due to quick loss of electrons from the trap. On the other hand if the power of RF field is $-100 \mathrm{dBm}$ then the excitation is very weak and the trapped cloud of electrons are slightly unstable, then some of the higher order axial oscillation resonances dips disappear and the intensities decrease with higher orders of resonances, as loss of electrons from the trap decreases. It can be observed from the Figure 3 (i), (ii) and (iv) that the axial oscillation frequency $f_{z}$ and its higher order multiples $2 f_{z}, 3 f_{z}, 4 f_{z}$ are observed at both the magnetic fields of $97 \mathrm{G}$ and $258 \mathrm{G}$. But the intensities of $f_{z}, 2 f_{z}, 3 f_{z}$ and $4 f_{z}$ decreased with decrease in power of $\mathrm{RF}$ field. But the modified cyclotron frequency and the side band frequency are almost remaining unchanged.

(ii) Storage voltage dependence: On comparing Fig.3 (iii) and (i) we can observe that, as the storage voltage is increased, the dips corresponding to axial resonances shift towards the region of higher frequencies of resonances spectra but the dips corresponding to cyclotron frequency and the side band frequency resonances remain unchanged. As the axial frequency and storage voltage are related as $f_{z} \propto \sqrt{V_{0}}\left(f_{z}=\frac{1}{2 \pi} \sqrt{\frac{4 q V_{0}}{m d^{2}}}\right)$, the shifts in dips corresponding to axial resonances are directly proportional to square root of storage voltages. But the pure cyclotron frequency is independent of storage voltage and hence the dips corresponding to cyclotron frequency and side band frequency $\left(f_{c}=f_{c}^{\prime}+f_{m}\right)$ resonances remain unchanged. 
Excitation frequency $(\mathrm{MHz})$

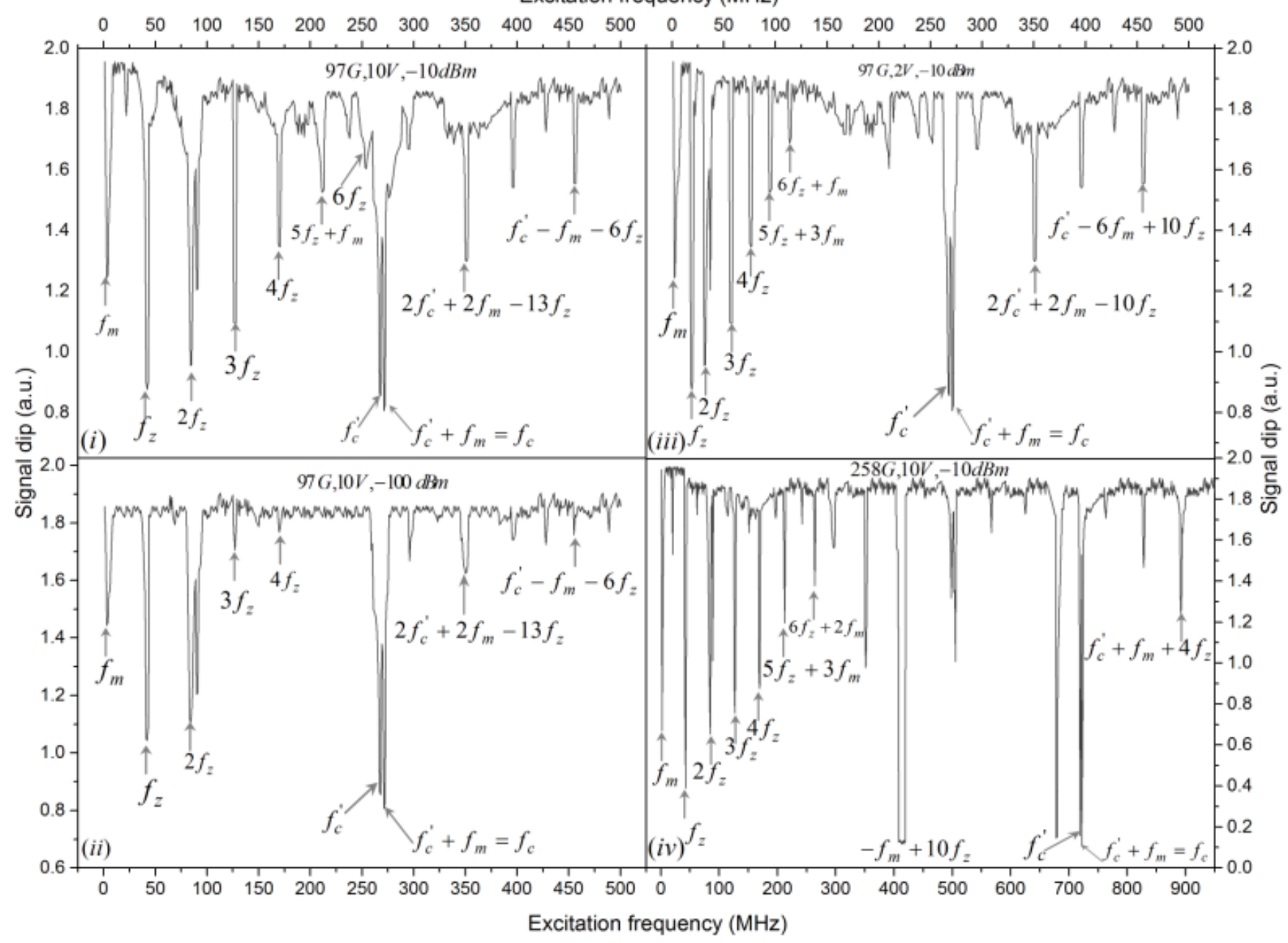

Figure 3: Motional resonances spectra showing dips for different magnetic fields, storage voltages and RF field powers of (i) $97 \mathrm{G}, 10 \mathrm{~V},-10 \mathrm{dBm}$ (ii) $97 \mathrm{G}$, 10V, $-100 \mathrm{dBm}$ (iii) $97 \mathrm{G}, 2 \mathrm{~V},-10 \mathrm{dBm}$, (iv) $258 \mathrm{G}, 10 \mathrm{~V},-10 \mathrm{dBm}$ [11]

(iii) Magnetic field dependence: The resonances spectra show the dependence on magnetic fields as observed in Figure 3 (i) at $97 \mathrm{G}$ and (iv) at $258 \mathrm{G}$. The axial oscillation frequency $f_{z}$ and its higher order multiples $2 f_{z}, 3 f_{z}, 4 f_{z}$ are observed at both the magnetic fields of $97 \mathrm{G}$ and $258 \mathrm{G}$. The axial frequency $\left(f_{z}\right)$ is independent of magnetic field, but the modified cyclotron frequency and magnetron frequency show dependence on the magnetic field.

\section{Oscillators models}

(i) Non-linear driven and damped harmonic oscillator model: We can develop a model of non-linear driven and damped harmonic oscillator for cloud of trapped electrons at very low RF field power of $-100 \mathrm{dBm}$ as shown in Figure $4[13,14]$. Non-linear spring is one that exerts a variable rate of force per unit displacement due to extension. A nonlinear spring has a defined non-linear loaddisplacement function, being equivalent to strain energy absorption rate of the spring. This means that the load applied to the spring is not proportional to the amount of extension produced, based on the spring's stretching rate as it is variable. A barrel spring's stretching rate is not a constant and whose force is based on a variable increase per unit distance of travel due to the different outer diameters per coil. The barrel spring's pitch is highly involved in spring's force, travel capacities and so having a variation of pitch between spring's coils will definitely alter springs force, turning this into a non-linear spring [14]. The equation of motion is given by

$$
M \ddot{z}+\alpha \dot{z}-F_{\text {restore }}=F_{0} z \sin \omega t
$$

The response at the third harmonic is generated by the cubic component of the restoring force $\left(F_{\text {restore }}\right)$. For a sinusoidal displacement $z=z_{0}(\cos \omega t+i \sin \omega t)=z_{0} e^{i \omega t}$, the restoring force is given by [14]

$$
F_{\text {restore }}=-M \omega_{z}^{2} z-\beta z^{3}=-M \omega_{z}^{2} z_{0} e^{-i \omega t}-\beta z_{0}^{3} e^{i 3 \omega t}
$$

With sinusoidal RF drive at frequency $f$, this non-linear cloud of electrons will respond at both the drive frequency $f$, and at the third harmonic of the drive frequency generating the third harmonic $3 f$. For non-linearities at the 


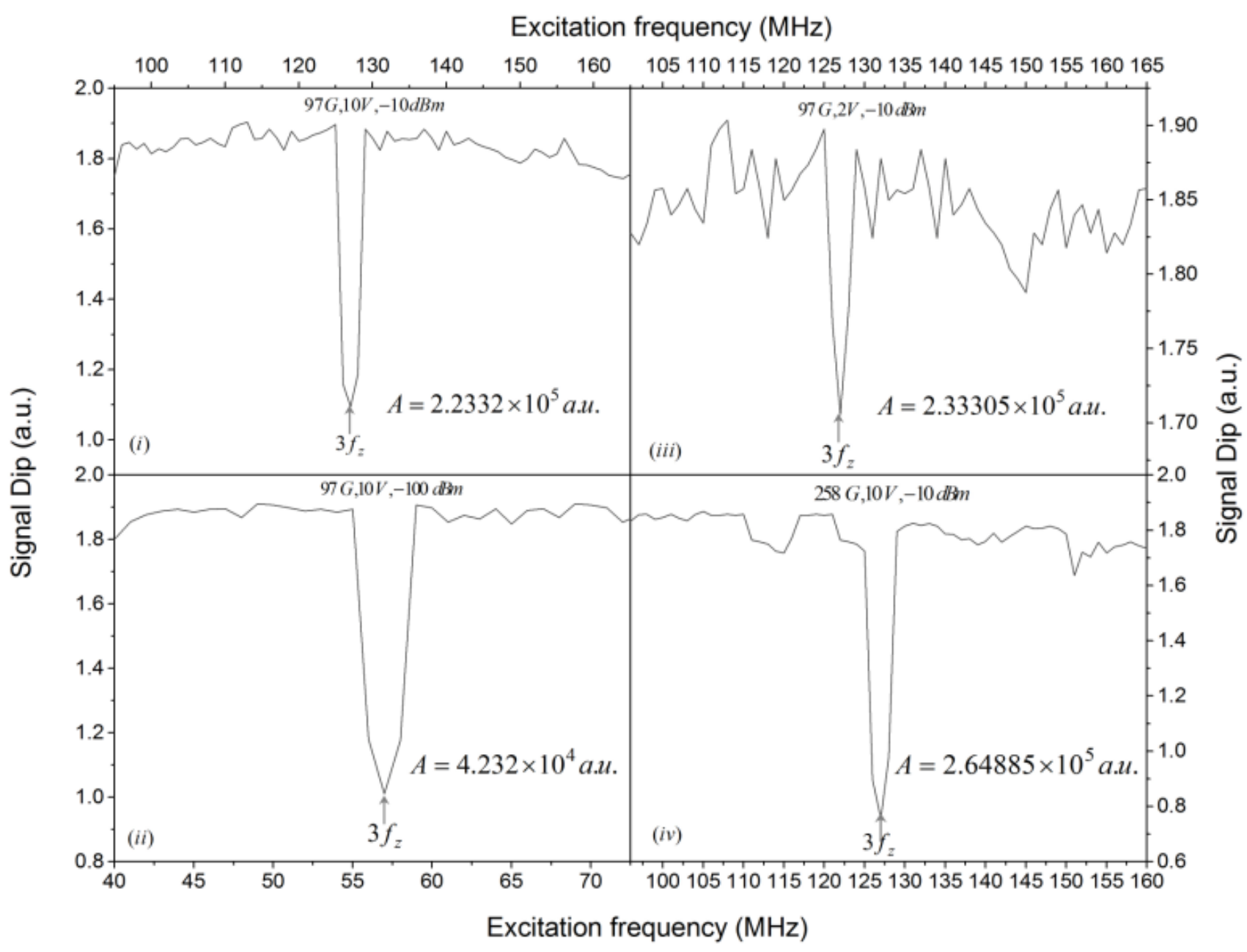

Figure 4: The strengths of 3rd harmonics in Motional resonances spectra for different magnetic fields, storage voltages and RF field powers of (i) $97 \mathrm{G}$, $10 \mathrm{~V}$,-10dBm (ii) $97 \mathrm{G}, 10 \mathrm{~V},-100 \mathrm{dBm}$ (iii) $97 \mathrm{G}, 2 \mathrm{~V},-10 \mathrm{dBm}$, (iv) $258 \mathrm{G}, 10 \mathrm{~V},-10 \mathrm{dBm}$

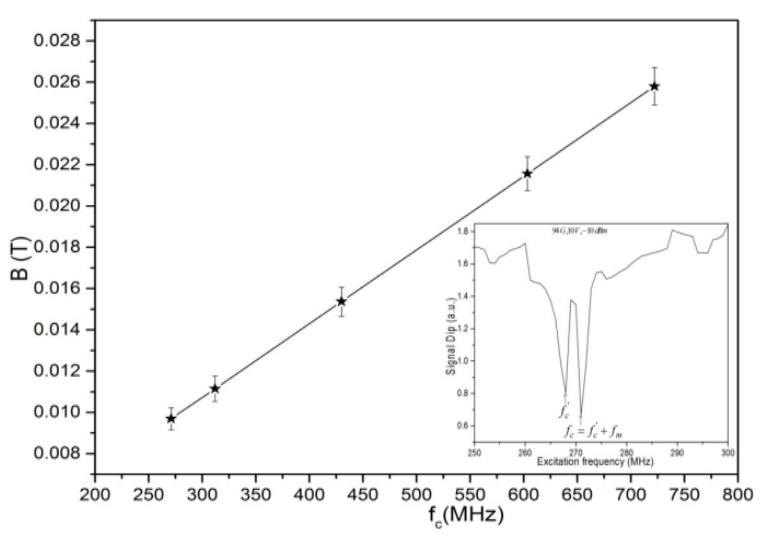

Figure 5: Magnetic field at the trap centre measured from cyclotron frequency from Motional resonances spectra

higher powers of $z^{n}$, the trapped cloud of electrons will generate the $n^{\text {th }}$ harmonic at frequency $n f$. When we drive the simple oscillator with a sinusoidal driving force,

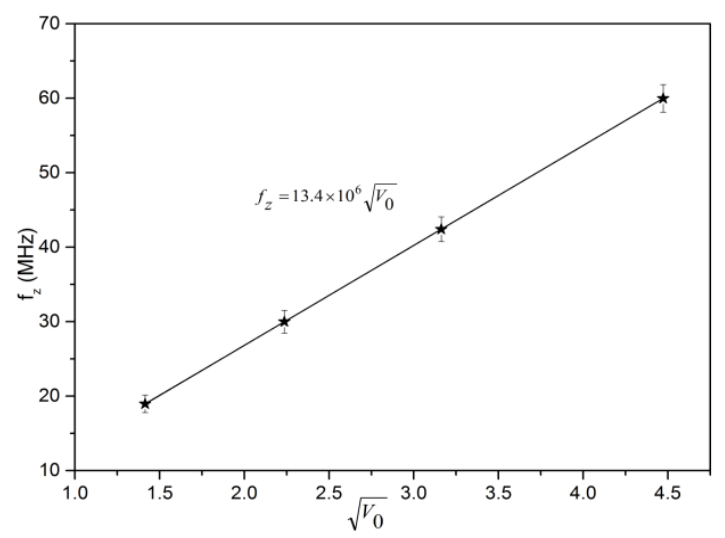

Figure 6: Axial resonance frequency versus square root of storage voltage

we get a response at the driving frequency. Suppose that we drive a nonlinear system with a frequency $f_{z}$, the output consists of the original frequency $f_{z}$ and its harmonics $2 f_{z}, 3 f_{z}, 4 f_{z}$, etc. The presence of harmonics depends on 


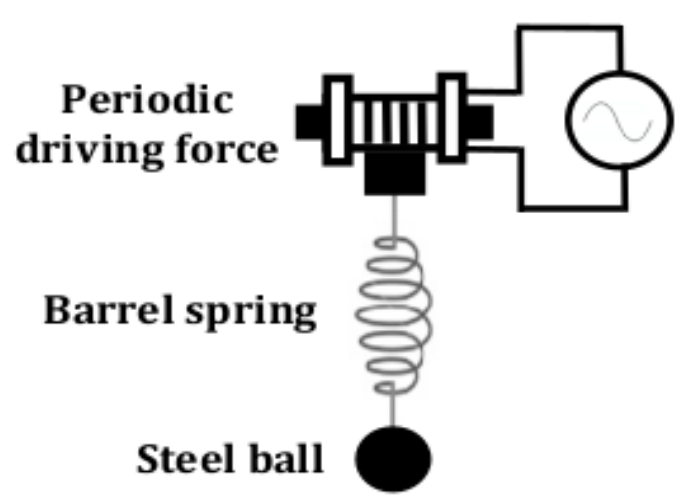

Figure 7: A periodically forced barrel spring with a steel ball at the free end can be regarded as a model of non-linear oscillator

the non-linearity in the cloud of electrons. The set of frequencies which are related to each other by integer ratios as $f_{n}=n f_{z}$ is called the harmonic series [15].

(ii) Driven Duffing oscillator model: We can develop a model of certain forced and damped non-linear oscillator described by non-linear second-order differential equation named after George Duffing as Duffing oscillator, for high RF field power of $-10 \mathrm{dBm}$ as shown in Figure $5[16,17]$. The forced Duffing oscillator is a system for the study of chaotic dynamics and development of analytical and experimental techniques for nonlinear systems. The doublewell Duffing oscillator model considered here is a narrow ferromagnetic flexible rod whose one end is fixed to a rigid support attached to a driving system and the free end carrying a steel ball, and positioned between two permanent magnets while undergoing lateral forces. This mechanical system has several features of the model Duffing oscillator such as a potential for the lateral position of the ball created by the two permanent magnets; nonautonomous forcing resulting in motion of the ball, potentially chaotically between the two wells; and mechanical dissipation within the ball and also from air resistance against the motion of ball $[18,19]$. The non-linear oscillations arise from a forced and narrow flexible rod with steel ball-magnets system, moving in the magnetic fields of two permanent magnets. As the flexible rod with steel ball is excited with an AC current, a cubic magnet-position dependent force appears on it. The magnet-flexible rod with steel ball interactions, results in the non-linear equation of motion similar to trapped electrons, this is found to be a forced and damped cubic Duffing oscillator moving in a quartic potential [20]. The motion of centre of mass of the cloud of electrons is described by non-linear second-order Duffing differential equation $[14,15]$

$$
M \ddot{z}+\alpha \dot{z}+M \omega_{z}^{2} z+\beta z^{3}=F_{0} z \sin \omega t
$$

The symbols $\alpha, \beta$, and $\omega$ are constants such that $\alpha$ is damping coefficient, $\beta$ is anharmonic coefficient and $\omega_{z}$ is harmonic coefficient. The appearance of the non-linear term $\beta z^{3}$ in Duffing equation changes the situation com-

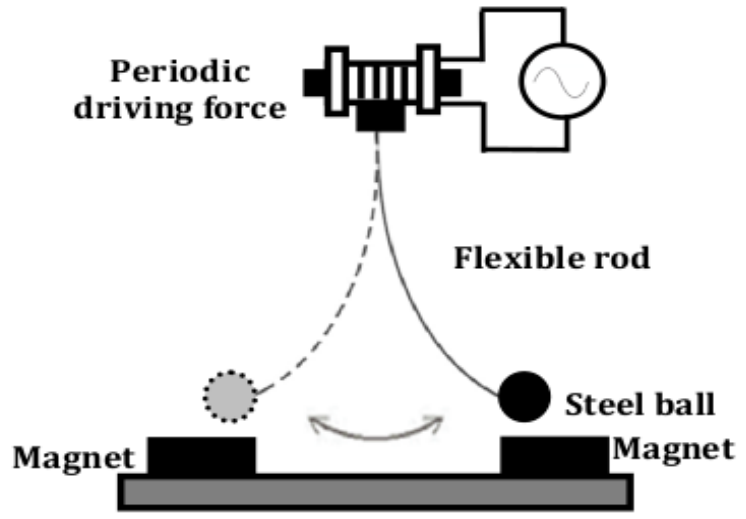

Figure 8: A periodically forced steel ball hung through a flexible rod, which is deflected alternately toward the two magnets can be regarded as a mechanical model of Duffing oscillator when the force is very strong [21]

pletely. For $\alpha>0$, the Duffing oscillator can be interpreted as a forced oscillator with a non-linear spring whose restoring force is written as [21] $F_{\text {restore }}=-\alpha z-$ $\beta z^{3}$. If $\beta>0$, the force is very strong, on the other hand if $\beta<0$, the force is very weak. The potential of timeindependent part of force $M \omega_{z}^{2} z+M \beta z^{3}$ is [22, 23]

$$
V(z)=\frac{1}{2} M \omega_{z}^{2} z^{2}+\frac{1}{4} M \beta z^{4}
$$

\section{Perturbative multipole terms in quadrupole potential}

Now consider the quadrupole potential in Penning trap on expanding equation (1) as

$$
\begin{aligned}
\phi(r, \theta) & =\phi_{0}\left[C_{0}+C_{1}\left(\frac{r}{r_{0}}\right)(\cos \theta)+C_{2}\left(\frac{r}{r_{0}}\right)^{2}+\frac{1}{3}[\cos 2 \theta-1]\right. \\
& +C_{3}\left(\frac{r}{r_{0}}\right)^{3} \frac{8}{5}[\cos 3 \theta-3 \cos \theta] \\
& \left.+C_{4}\left(\frac{r}{r_{0}}\right)^{4} \frac{192}{105}[\cos 4 \theta-80 \cos 2 \theta-7] \ldots \infty\right]
\end{aligned}
$$

The Legendre coefficients $C_{2}$ and $C_{4}$ represent the quadrupole term and octupole term respectively. Here $C_{2}$ represents the pure quadrupole term which can be normalized to 1 , and $C_{4}$ is the most likely leading term of the perturbation.

(i) Quadrupole moment co-efficient $\left(C_{2}\right)$ The quadrupole moment co-efficient $C_{2}$ can be calculated from axial frequency as $[8,11]$

$$
\omega_{z}=\sqrt{\frac{4 e V_{0}\left(1+C_{2}\right)}{m\left(r_{0}^{2}+2 z_{0}^{2}\right)}}, \quad C_{2}=\frac{4 \pi f_{z}^{2} m\left(r_{0}^{2}+2 z_{0}^{2}\right)}{4 e V_{0}}-1
$$

If $f_{z}=10 \times 10^{6} \mathrm{~Hz}$ and $V_{0}=0.7 \mathrm{~V}$, then $C_{2}=-0.2$. From Figure $6, C_{2}$ value can be calculated from slope $13.4 \times 10^{6}$ of cyclotron frequency $v / s$ square root of trap voltage

$$
\frac{f_{z}}{\sqrt{V_{0}}}=13.4 \times 10^{6}
$$




$$
C_{2}=\frac{\pi^{2} m\left(r_{0}^{2}+2 z_{0}^{2}\right)}{e}\left[\frac{f_{z}}{\sqrt{V_{0}}}\right]^{2}-1=-0.1
$$

\section{(ii) Octupole moment co-efficient $\left(C_{4}\right)$}

The octupole moment co-efficient $C_{4}$ can be measured from the width of the axial resonance line. If $E_{z}$ is the energy in axial direction, $f_{z}$ is the axial frequency of oscillations, $R=5 \mathrm{~mm}$ is the amplitude of the axial oscillations, we consider $R$ to be the radius of the electron cloud with the identical resonant frequency $[9,11]$.

$$
\Delta \omega_{z} \approx \frac{3}{2} \frac{C_{4} E_{z} \omega_{z}}{e V_{0}}, C_{4} \approx \frac{e V_{0}}{6 \pi E_{z} f_{z}} \Delta \omega_{z}
$$

If $f_{z}=10 \times 10^{6} \mathrm{~Hz}$ and $V_{0}=0.7 \mathrm{~V}$, then $E_{z}=\frac{1}{2} m_{e} \omega_{z}^{2} E_{e c}^{2}=$ $4.486 \times 10^{-20} \mathrm{~J}$. Therefore $C_{4} \approx 0.032$.

Table 2: $C_{2}$ and $C_{4}$ simulation values for trap design and experimental values

\begin{tabular}{lcll}
\hline$C_{n}$ & Equation & $\begin{array}{l}\text { Trap design } \\
\text { values simu- } \\
\text { lation }\end{array}$ & $\begin{array}{l}\text { Experimental } \\
\text { values }\end{array}$ \\
\hline$C_{2}$ & $\frac{4 \pi f_{z}^{2} m\left(r_{0}^{2}+2 z_{0}^{2}\right)}{4 e V_{0}}-1$ & -0.17 & -0.2 \\
\hline$C_{4}$ & $\frac{e V_{0}}{6 \pi E_{z} f_{z}} \Delta \omega_{z}$ & 0.03 & 0.032 \\
\hline
\end{tabular}

\section{Conclusion}

The non-linear higher order motional resonances of the cloud of electrons are observed, that result from couplings in the motional degrees of freedom and characterize the perturbations in the trap. The likely presence of perturbative terms of quadrupole potential is identified by studying the motional resonances spectra and hence Quadrupole moment co-efficient $\left(C_{2}\right)$, Octupole moment co-efficient $\left(C_{4}\right)$ multipole terms are calculated. The resonances spectra show the dependence on magnetic fields, RF field powers and storage voltages. A periodically forced barrel spring with a steel ball at the free end can be regarded as a model of non-linear oscillator and a periodically forced steel ball hung through a flexible rod, which is deflected alternately toward the two magnets can be regarded as a mechanical model of Duffing oscillator are developed for the observation of non-linear higher order axial oscillatory motional resonances that consist of the original frequency $f_{z}$ and its harmonics $2 f_{z}, 3 f_{z}, 4 f_{z}$, etc and the presence of harmonics depend on the non-linearity in the cloud of trapped electrons, for different RF field excitation powers of $-10 \mathrm{dBm}$ and $-100 \mathrm{dBm}$, respectively.

\section{Appendix}

MATLAB Code for generation of all possible integer triplets [11] for $K=0.02$

$\mathrm{A} 1=0.0220$;

$\mathrm{B} 1=0.0218$;

$\mathrm{A} 2=0.0220$;

$\mathrm{B} 2=0.0218$; $\mathrm{h}=1$;

for $\mathrm{nc}=0: 1: 5$

for $\mathrm{nm}=-10: 1: 10$

for $\mathrm{nz}=-10: 1: 10$

$\mathrm{A}=2 *(\mathrm{nc}-\mathrm{nm}) \wedge 2+4 * \mathrm{nz} \wedge 2$;

$\mathrm{B}=-2 *(\mathrm{nc}+\mathrm{nm}) * \mathrm{nz}$;

$\mathrm{C}=4 * \mathrm{nc} * \mathrm{~nm}$;

$\mathrm{L}=\operatorname{abs}(\mathrm{nz})+\operatorname{abs}(\mathrm{nc})+\mathrm{abs}(\mathrm{nm})$;

if $(A>0)$

if $\left(\left(B^{\wedge} 2-A * C\right)>=0\right)$

$\mathrm{K} 1=\left(\mathrm{B}+\operatorname{sqrt}\left(\mathrm{B}^{\wedge} 2-\mathrm{A} * \mathrm{C}\right)\right) / \mathrm{A}$;

$\mathrm{K} 2=\left(\mathrm{B}-\operatorname{sqrt}\left(\mathrm{B}^{\wedge} 2-\mathrm{A} * \mathrm{C}\right)\right) / \mathrm{A}$;

if $((K 1<=0.022) \& \&(K 1>0.018))||((K 2<=0.022)$

$\& \&(K 2>0.018))$

$\operatorname{char}(\mathrm{h},:)=[\mathrm{nc}, \mathrm{nm}, \mathrm{nz}, \mathrm{L}, \mathrm{K} 1]$;

$\mathrm{h}=\mathrm{h}+1$;

end

end

end

end

end

end

\section{Acknowledgement}

I acknowledge Prof. Sharath Ananthamurthy, Department of Physics, Bangalore University, Bengaluru, for the support to carrying out experiment and Bashyam Govindan, Chennai, India, who helped to develop MATLAB Code for generation of all possible integer triplets for desired values of $\mathrm{K}$ and Reviewers of JNSPS who assisted immensively in improving this article.

\section{References}

[1] P. Paasche, T. Valenzuela, D. Biswas, C. Angelescu, \& G. Werth , "Individual and center of mass resonances in the motional spectrum of an electron cloud in a Penning trap" Eur. Phys. J. D 18 (2002) 295.

[2] P. Paasche, C. Anglescu, S. Ananthamurthy, D. Biswas, T. Valenzuala, G. Werth, "Instabilities of an electron cloud in a Penning trap", Eur. Phys. J. D 22 (2003) 183.

[3] G. Tommaseo, P. Paasche, C. Angelescu, \& G. Werth, "Subharmonic excitation of the eigenmodes of charged particles in a Penning trap", Eur. Phys. J. D 28 (2004) 39.

[4] A. Drakoudis, A. M. Sallner, \& A. G. Werth, "Instabilities of ion motion in a linear Paul trap", Int. J. Mass Spectrom. 252 (2006) 61.

[5] X. Z. Chu, M. Holzki, R. Alheit, \& G. Werth, "International Journal of mass spectrometry and Ion Processes" 173 (1998) 107.

[6] R. Alheit, S. Kleineidam, F. Vedel, M. Vedel, \& G. Werth, "International Journal of mass spectrometry and Ion Processes" 154 (1996) 155.

[7] F. G. Major, V. N. Gheorghe, \& G. Werth, "Charged Particle Traps, Physics and techniques of charged particle confinement", (Springer) (2005).

[8] P. K.Ghosh, "Ion Traps", (Clarendon Press, Oxford ) (1995) 72.

[9] K. T. Satyajit, D. Datar \& S. Ananthamurthy, "Electron Storage Instabilities and Resonances in a Quadrupole Penning Trap", Asian Journal of Physics 19 (2010) 1

[10] M. Kretzschmar, "Single particle motion in a penning trap: description in the classical canonical formalism”. Physica Scripta 46 (1992) 544.

[11] B. M. Dyavappa, "Spectroscopy of non-neutral plasmas in ion traps", Motional Resonances Spectra of electrons, (Ph.D. thesis, Bangalore University, (2017).

[12] K. T. Sathyajith, "Spectroscopy of Electrons and ions in Electromagnetic Traps", Ph.D. thesis, Bangalore University, (2010). 
[13] Y. Ueda, "Randomly transitional phenomena in the system governed by Duffing's Equation", Journal of Statistical Physics 20 (1979) 181.

[14] "Driven Nonlinear Oscillators", Physics 15a Lab, (Spring) (2013).

[15] B. . Roberts, "Notes on Linear and Nonlinear Oscillators, and Periodic Waves," Department of Physics, Boston University, PY231 (2011).

[16] "Maple worksheets on the Duffing equation", http://www.peterstone.name/Maplepgs/ duffing. html.

[17] T. Kanamaru, "Duffing Oscillator", Scholarpedia 3 (2008) 6327.

[18] A. Champion, R. Granowski, A. Lodhi, \& S. Ravi. "Dynamics of a Periodically Forced Duffing Oscillator with Asymmetric Potential" (2012).

[19] "Bistable Duffing Oscillator with Piezoelectric Coupling: The Piezomagneto-elastic Energy Harvester, Piezoelectric energy harvesting, New and Renewable Energy Technologies for Sustainable Development",
(2004).

[20] G. Donoso \& C. L. Ladera, "Nonlinear dynamics of a magnetically driven Duffing-type spring-magnet oscillator in the static magnetic field of a coil", European Journal of Physics A 33 (2012).

[21] M. Rafikov, J. M. Balthazar, \& A. M. Tusset, "An optimal linear control design for nonlinear systems", J. Braz. Soc. Mech. Sci. \& Eng. 30 (2008) 1473.

[22] H. J. Korsch, H. J. Jodl, \& T. Hartmann, "Chaos, A Program Collection for the PC", 3rd edition, (Springer) (2008).

[23] M. Rafikov, J. M. Balthazar, A. M. Tusset, \& J. Braz. "An optimal linear control design for nonlinear systems”, Soc. Mech. Sci. \& Eng. 30 (2008). 